\title{
Disjoint hypercyclic operators
}

\author{
by \\ Luis BERnAL-GonZÁLEZ (Sevilla)
}

\begin{abstract}
We introduce the concept of disjoint hypercyclic operators. These are operators performing the approximation of any given vectors with a common subsequence of iterates applied on a common vector. The notion is extended to sequences of operators, and applied to composition operators and differential operators on spaces of analytic functions.
\end{abstract}

1. Introduction. In the last 25 years there have been important advances in the research of hypercyclicity, from several points of view. Roughly speaking, hypercyclicity means existence of a dense orbit. In this paper, we are concerned with the existence of a common vector with dense orbit for several operators, such that approximation of any fixed vectors is also simultaneously performed by using a common subsequence. This will be formalized later. Although we have been inspired by an interesting work about universal Taylor series due to Costakis and Vlachou [20], the path of research opened in the current paper is, as far as we know, new. Below, we fix some related notation and terminology to be used in the present article. For a good account of concepts, results and history concerning hypercyclicity, the reader is referred to the surveys [26], [28] and [15].

By $\mathbb{N}, \mathbb{N}_{0}, \mathbb{R}, \mathbb{C}, \mathbb{D}, B(a, r), \bar{B}(a, r)(a \in \mathbb{C}, r>0)$ we denote, respectively, the set of positive integers, the set $\mathbb{N} \cup\{0\}$, the real line, the complex plane, the open unit disk $\{z \in \mathbb{C}:|z|<1\}$, the open disk with center $a$ and radius $r$, and the corresponding closed disk. Assume that $X$ and $Y$ are two (Hausdorff) topological vector spaces over $\mathbb{K}=\mathbb{R}$ or $\mathbb{C}$. Then $L(X, Y)$ will stand for the space of all continuous linear mappings $T: X \rightarrow Y$. In

2000 Mathematics Subject Classification: Primary 47A16; Secondary 30E10, 47B33, 47B38.

Key words and phrases: hypercyclic operator, hypercyclic sequence, simultaneous approximation, composition operator, differential operator.

The author has been partially supported by the Plan Andaluz de Investigación de la Junta de Andalucía FQM-127 and by MEC Grants MTM2006-13997-C02-01 and Acción Especial MTM2004-21420-E. 
particular, if $X=Y$, then $L(X):=L(X, X)=$ \{operators on $X\}$. If $\left(T_{n}\right)$ is a sequence in $L(X, Y)$, then $\left(T_{n}\right)$ is said to be hypercyclic whenever there exists a vector $x \in X$-called a hypercyclic vector for $\left(T_{n}\right)$-whose orbit $\left\{T_{n} x: n \in \mathbb{N}\right\}$ under $\left(T_{n}\right)$ is dense in $Y$ (so $Y$ is necessarily separable). We denote by $\operatorname{HC}\left(\left(T_{n}\right)\right)$ the set of hypercyclic vectors for $\left(T_{n}\right)$. An operator $T \in L(X)$ is said to be hypercyclic provided that the sequence $\left(T^{n}\right)$ of iterates of $T$ is hypercyclic. In this case the set $\operatorname{HC}(T):=\operatorname{HC}\left(\left(T^{n}\right)\right)$ is called the set of hypercyclic vectors for $T$. If $\operatorname{HC}\left(\left(T_{n}\right)\right)$ is dense in $X$, then $\left(T_{n}\right)$ is said to be densely hypercyclic. Finally, recall that an $F$-space is a completely metrizable topological vector space.

Assume that $X, Y, Z$ are separable F-spaces, and that $S_{n}: X \rightarrow Y$ and $T_{n}: X \rightarrow Z(n \in \mathbb{N})$ are densely hypercyclic sequences. Since $\operatorname{HC}\left(\left(S_{n}\right)\right)$, $\mathrm{HC}\left(\left(T_{n}\right)\right)$ are dense $G_{\delta}$ subsets of $X$, it follows that $\mathrm{HC}\left(\left(S_{n}\right)\right) \cap \mathrm{HC}\left(\left(T_{n}\right)\right)$ is also dense, so nonempty. Hence there is a common hypercyclic vector $x \in X$. So, for any given vectors $y \in Y, z \in Z$, there are sequences $\left\{n_{1}<n_{2}<\cdots\right\}$ and $\left\{m_{1}<m_{2}<\cdots\right\}$ in $\mathbb{N}$ such that $S_{n_{j}} x \rightarrow y$ and $T_{m_{j}} x \rightarrow z$ as $j \rightarrow \infty$. Now, we pose the following question (a similar conclusion as above can be obtained and a similar question can be posed for single hypercyclic operators on $X$, just by considering the sequences of their iterates):

Under what conditions on $\left(S_{n}\right)$ and $\left(T_{n}\right)$ can one guarantee the existence of a vector $x \in X$ such that, for any given $y \in Y$ and $z \in Z$, there is one sequence $\left\{n_{1}<n_{2}<\cdots\right\} \subset \mathbb{N}$ such that $S_{n_{j}} x \rightarrow y$ and $T_{n_{j}} x \rightarrow z(j \rightarrow \infty)$ ?

Motivated by the last question, the new concepts of jointly hypercyclic operators and of disjoint hypercyclic sequences of continuous linear mappings are introduced in Section 2. Also, two workable sufficient conditions for disjoint hypercyclicity will be provided in the setting of F-spaces (see Section 4). Before this, we introduce in Section 3 two new notions: supermixing operators and sequences of operators controlled by seminorms. Finally, in Section 5, the disjoint hyperciclity conditions will be applied on spaces of analytic functions to identify finite sets of sequences of either infinite order differential operators or composition operators that are disjoint hypercyclic.

2. Disjoint hypercyclic operators. Let us define the new concept that is the subject of this paper.

Definition 2.1. Let $p \in \mathbb{N}$ and $X, Y_{1}, \ldots, Y_{p}$ be topological vector spaces. Assume that, for each $j \in\{1, \ldots, p\}, T_{j, n}: X \rightarrow Y_{j}(n \in \mathbb{N})$ is a sequence of continuous linear mappings. We say that the sequences $\left(T_{1, n}\right), \ldots,\left(T_{p, n}\right)$ are disjoint hypercyclic if the sequence $\left[T_{1, n}, \ldots, T_{p, n}\right]$ : 
$X \rightarrow Y_{1} \times \cdots \times Y_{p}(n \in \mathbb{N})$ defined as

$$
\left[T_{1, n}, \ldots, T_{p, n}\right] x=\left(T_{1, n} x, \ldots, T_{p, n} x\right) \quad(x \in X)
$$

is hypercyclic, where $Y_{1} \times \cdots \times Y_{p}$ is assumed to be endowed with the product topology. We say that the sequences $\left(T_{1, n}\right), \ldots,\left(T_{p, n}\right)$ are densely disjoint hypercyclic if the sequence $\left(\left[T_{1, n}, \ldots, T_{p, n}\right]\right)$ is densely hypercyclic. If $T_{1}, \ldots, T_{p} \in L(X)$ then $T_{1}, \ldots, T_{p}$ are said to be disjoint hypercyclic (densely disjoint hypercyclic, resp.) provided that the sequences $\left(T_{1}^{n}\right), \ldots,\left(T_{p}^{n}\right)$ are disjoint hypercyclic (densely disjoint hypercyclic, resp.).

Remarks 2.1. 1. Clearly, the disjoint hypercyclicity of $\left(T_{1, n}\right), \ldots,\left(T_{p, n}\right)$ remains unaltered under a change of order $\left(T_{\sigma(1), n}\right), \ldots,\left(T_{\sigma(p), n}\right)$. Also, trivially, it implies the hypercyclicity of each sequence $\left(T_{j, n}\right)(j=1, \ldots, p)$.

2. Let $X, Y, Z$ be separable F-spaces. According to [26], being densely hypercyclic is equivalent to being topologically transitive, in the sense of Birkhoff. Thus, saying that two sequences $\left(S_{n}\right) \subset L(X, Y),\left(T_{n}\right) \subset L(X, Z)$ are densely disjoint hypercyclic is the same as saying that if $U, V, W$ are nonempty open subsets of $X, Y, Z$, respectively, there exists an $n$ (equivalently, there exist infinitely many $n)$ such that $U \cap S_{n}^{-1}(V) \cap T_{n}^{-1}(W) \neq \emptyset$.

3. The mere hypercyclicity of two sequences $\left(S_{n}\right),\left(T_{n}\right)$ does not imply their disjoint hypercyclicity: take $\left(T_{n}\right)$ hypercyclic and $\left(S_{n}\right):=\left(T_{n}\right)$.

4 . The reader should be warned not to confuse the disjoint hypercyclicity of $\left(T_{1, n}\right), \ldots,\left(T_{p, n}\right)$ with the hypercyclicity of the sequence $T_{1, n} \oplus \cdots \oplus T_{p, n}:\left(x_{1}, \ldots, x_{p}\right) \in X^{p} \mapsto\left(T_{1, n} x_{1}, \ldots, T_{p, n} x_{p}\right) \in Y_{1} \times \cdots \times Y_{p}$.

Disjoint hypercyclicity implies the hypercyclicity of the last sequence, but the converse is false.

5. It is well known that $\left(T^{n}\right)$ is densely hypercyclic as soon as $T$ is hypercyclic. But, in principle, nothing guarantees the dense hypercyclicity of the sequence $\left(\left[S^{n}, T^{n}\right]\right)$ if $S, T$ are disjoint hypercyclic operators. Therefore we pose the problem of whether there exist disjoint hypercyclic operators $S, T$ such that $\left(\left[S^{n}, T^{n}\right]\right)$ is not densely disjoint hypercyclic. For this reason, we have been forced in Definition 2.1 to define the dense disjoint hypercyclicity for several single operators, which would be superfluous for one operator.

6 . In spite of the last remark, we have found a situation where the answer to the problem just posed is affirmative. It is inspired by [24, Section 4] and [28, Section 2]. Recall that if $T$ is a hypercyclic operator on a topological vector space $X$, then $\mathrm{HC}(T)$ contains, except for zero, a dense linear submanifold (in the terminology of [2], $\mathrm{HC}(T)$ is algebraically generic in $X$ ); see [10], [17], [30] and [41]. In this order of ideas, we give the following statement, which can be easily proved just by taking into account (see [41]) that $P(T)$ has dense range if $T$ is hypercyclic and $P$ is a nonzero polynomial with 
coefficients in $\mathbb{K}$ : Suppose that $T_{1}, \ldots, T_{p}$ are disjoint hypercyclic operators on a topological vector space $X$. Assume also that there is $i \in\{1, \ldots, p\}$ such that every $T_{j}$ commutes with $T_{i}$. Then the sequence $\left(\left[T_{1}^{n}, \ldots, T_{p}^{n}\right]\right)$ is densely hypercyclic. Even more, $\mathrm{HC}\left(\left(\left[T_{1}^{n}, \ldots, T_{p}^{n}\right]\right)\right)$ is algebraically generic in $X$.

3. Control by seminorms and supermixing operators. In order to isolate the properties that will be needed, we introduce two concepts concerning operators defined on Fréchet spaces. Recall that an F-space $X$ is called a Fréchet space whenever $X$ is locally convex. The family of continuous seminorms on such a space $X$ will be denoted by $\operatorname{SN}(X)$. If $p \in \mathrm{SN}(X)$, then we define a $p$-open set of $X$ as a union of open $p$-balls $\{x \in X: p(x-\alpha)<\varepsilon\}(\alpha \in X, \varepsilon>0)$.

Definition 3.1. Let $X, Y$ be Fréchet spaces, and $\left(T_{n}\right)$ be a sequence in $L(X, Y)$. We say that $\left(T_{n}\right)$ is controlled by seminorms, or that it is $S N$ controlled, if for every $q \in \mathrm{SN}(Y)$ there are $p \in \mathrm{SN}(X)$ and constants $C_{n} \in(0, \infty)(n \in \mathbb{N})$ such that $q\left(T_{n} x\right) \leq C_{n} p(x)$ for all $x \in X$ and all $n \in \mathbb{N}$. Equivalently, for every $q \in \mathrm{SN}(X)$ there is $p \in \operatorname{SN}(X)$ such that $T_{n}^{-1}(V)$ is a $p$-open set in $X$ for each $q$-open set $V$ in $Y$ and each $n \in \mathbb{N}$.

REMARK 3.1. The point in the above definition is that $p$ depends on $q$, but not on $n$. Observe that if $\left(T_{n}\right)$ is equicontinuous then $\left(T_{n}\right)$ is SNcontrolled (with a common constant $C_{n}=C$ for all $n$ ). Between Banach spaces $X$ and $Y$, any sequence $\left(T_{n}\right) \subset L(X, Y)$ is SN-controlled. Hence the $\mathrm{SN}$-control is only of interest for nonnormable Fréchet spaces.

Before giving some examples, let us fix some additional notation. By a domain we mean a nonempty connected open subset $G \subset \mathbb{C}$. Then $H(G)$ denotes the Fréchet space of holomorphic functions in $G$ endowed with the topology of uniform convergence on compacta. It is known that $H(G)$ is separable and nonnormable. If $G=\mathbb{C}$, then we obtain in particular the space $\mathcal{E}=H(\mathbb{C})$ of entire functions.

Let $\Phi(z)=\sum_{n=0}^{\infty} a_{n} z^{n} \in \mathcal{E}$. Then $\Phi$ is said to be of exponential type (the set of these functions will be denoted by $\operatorname{Exp}$ ) provided that there are positive constants $A, B$ such that $|\Phi(z)| \leq A \exp (B|z|)$ for all $z \in \mathbb{C}$. Consider its associated formal linear (in general, infinite order) differential operator $\Phi(D)=\sum_{n=0}^{\infty} a_{n} D^{n}$ defined as $\Phi(D) f=\sum_{n=0}^{\infty} a_{n} f^{(n)}(f \in \mathcal{E})$. Then $\Phi(D)$ is a well-defined operator on $\mathcal{E}$. Moreover, an operator $T \in L(\mathcal{E})$ commutes with translations (that is, $T \circ \tau_{a}=\tau_{a} \circ T$ for all $a \in \mathbb{C}$, where $\left(\tau_{a} f\right)(z):=f(z+a)$ for $z \in \mathbb{C}$ and $\left.f \in \mathcal{E}\right)$ if and only if it commutes with the derivative operator $D\left(D f:=f^{\prime}\right)$, and if and only if there exists $\Phi \in \operatorname{Exp}$ such that $T=\Phi(D)$ (see [23, Section 5]). Note that $D$ and the translations $\tau_{a}$ are special cases (take $\Phi(z) \equiv z$ and $\Phi(z) \equiv e^{a z}$, resp.). 
Assume that $\Phi$ is an entire function of subexponential type, that is, for given $\varepsilon>0$ there is a constant $A>0$ such that $|\Phi(z)| \leq A \exp (\varepsilon|z|)$ for all $z \in \mathbb{C}$ (so, in particular, $\Phi \in \operatorname{Exp}$ ); equivalently, $\lim _{n \rightarrow \infty}\left(n !\left|a_{n}\right|\right)^{1 / n}=0$ (see [14]). Suppose that $G$ is any domain in $\mathbb{C}$. Then $\Phi(D)$ is an operator on $H(G)$, not only on $\mathcal{E}$ (see [3]).

\section{EXAMPLES 3.2.}

1. Suppose that $G$ is a domain in $\mathbb{C}$. If $\left(\Phi_{n}\right)$ is any sequence of entire functions of subexponential type, then the sequence $\left(T_{n}\right):=\left(\Phi_{n}(D)\right)$ is SN-controlled in $H(G)$. To see this, take a sequence of compact sets $\left(K_{k}\right)$ such that $G=\bigcup_{k=1}^{\infty} K_{k}, K_{k} \subset K_{k+1}^{\circ}(k \in \mathbb{N})\left(A^{\circ}\right.$ denotes the interior of a subset $A \subset \mathbb{C}$ ) and the boundary $\partial K_{k}$ of each $K_{k}$ is the union of finitely many rectifiable Jordan curves, which can be assumed to be oriented counterclockwise. It is even possible to construct $\left(K_{k}\right)$ so that, for every $k$, each bounded connected component of $\mathbb{C} \backslash K_{k}$ (i.e., each "hole" of $K_{k}$ ) contains some bounded connected component of $\mathbb{C} \backslash G$ (see [19] and [37]). The increasing sequence $\mathcal{P}:=\left(p_{k}\right)$ of seminorms given by

$$
p_{k}(f):=\sup \left\{|f(z)|: z \in K_{k}\right\} \quad(k \in \mathbb{N}, f \in H(G))
$$

defines the topology of $H(G)$. Fix a seminorm $q \in \mathrm{SN}(H(G))$. Then there are $\varepsilon>0$ and $k \in \mathbb{N}$ such that $q(f) \leq \varepsilon p_{k}(f)$ for all $f \in H(G)$. Let $\Phi_{n}(z)=\sum_{j=0}^{\infty} a_{j, n} z^{j}(z \in \mathbb{C}, n \in \mathbb{N})$. Since $\left(j !\left|a_{j, n}\right|\right)^{1 / j} \rightarrow 0(j \rightarrow \infty)$, there is a constant $A_{n}>0$ such that $j !\left|a_{j, n}\right| \leq A_{n} \varepsilon_{1}^{j}\left(j \in \mathbb{N}_{0}\right)$, where $\varepsilon_{1}:=(1 / 2) \operatorname{dist}\left(K_{k}, \partial K_{k+1}\right)$. Let $p:=p_{k+1}$ and

$$
C_{n}:=\frac{\varepsilon A_{n} \operatorname{length}\left(\partial K_{k+1}\right)}{\operatorname{dist}\left(K_{k}, \partial K_{k+1}\right)} \quad(n \in \mathbb{N}) .
$$

Fix $f \in H(G)$. Then the Cauchy integral formula for derivatives shows that, for every $z \in K_{k}$,

$$
\begin{aligned}
\left|T_{n} f(z)\right| & =\left|\sum_{j=0}^{\infty} a_{j, n} f^{(j)}(z)\right|=\left|\sum_{j=0}^{\infty} \frac{a_{j, n} j !}{2 \pi i} \oint_{\partial K_{k+1}} \frac{f(t)}{(t-z)^{j+1}} d t\right| \\
& \leq \sum_{j=0}^{\infty} \frac{A_{n} \varepsilon_{1}^{j}}{2 \pi} \frac{\sup _{t \in \partial K_{k+1}}|f(t)| \text { length }\left(\partial K_{k+1}\right)}{\left(\operatorname{dist}\left(K_{k}, \partial K_{k+1}\right)\right)^{j+1}} \\
& \leq \frac{A_{n} \operatorname{length}\left(\partial K_{k+1}\right) \sup _{w \in K_{k+1}}|f(w)|}{2 \operatorname{dist}\left(K_{k}, \partial K_{k+1}\right)} \sum_{j=0}^{\infty}\left(\frac{1}{2}\right)^{j} \\
& =\frac{A_{n} \operatorname{length}\left(\partial K_{k+1}\right)}{\operatorname{dist}\left(K_{k}, \partial K_{k+1}\right)} p_{k+1}(f)=\frac{C_{n}}{\varepsilon} p(f) .
\end{aligned}
$$

Therefore, $q\left(T_{n} f\right) \leq \varepsilon p_{k}\left(T_{n} f\right) \leq \varepsilon \sup \left\{\left|T_{n} f(z)\right|: z \in K_{k}\right\} \leq C_{n} p(f)$, as desired. 
2. However, if the $\Phi_{n}$ are merely of exponential type, then the sequence $\left(\Phi_{n}(D)\right)(\subset L(\mathcal{E}))$ is not always $\mathrm{SN}$-controlled. For instance, the sequence $\left(\tau_{n}\right)$ of translations (case $\Phi_{n}(z)=e^{n z}$ ) is never SN-controlled. Indeed, let $q:=p_{1}$ and fix $p \in \operatorname{SN}(\mathcal{E})$ and $C_{n}>0(n \in \mathbb{N})$, where $p_{n}(f):=\sup \{|f(z)|:$ $|z| \leq n\}$. We wish to find a function $F \in \mathcal{E}$ and a positive integer $N$ such that $q\left(\tau_{N} F\right)>C_{N} p(F)$. Since $p$ is continuous, there exist $n \in \mathbb{N}$ and $\varepsilon>0$ satisfying $\{f \in \mathcal{E}: p(f)<1\} \supset\left\{f \in \mathcal{E}: p_{n}(f)<\varepsilon\right\}$. Since the balls $\bar{B}(0, n)$ and $\bar{B}(n+2,1)$ are disjoint, we can apply Runge's approximation theorem (see [22]) to obtain a polynomial $F$ (so $F \in \mathcal{E}$ ) such that $|F(z)-0|<\varepsilon$ on $\bar{B}(0, n)$ and $\left|f(z)-\left(C_{n+2}+1\right)\right|<1$ on $\bar{B}(n+2,1)$. Therefore $p(f)<1$ and $q\left(\tau_{n+2} F\right)>C_{n+2}$. Hence, for $N:=n+2$, we get $q\left(\tau_{N} F\right)>C_{N} p(F)$, as required.

The second new concept that will be introduced has to do with the "improvement of quality" of the hypercyclicity. First, let us recall a number of related notions. Assume that $X, Y$ are separable F-spaces and that $\left(T_{n}\right) \subset$ $L(X, Y)$. Then $\left(T_{n}\right)$ is said to be weakly mixing whenever the sequence

$$
T_{n} \oplus T_{n}:(x, y) \in X^{2} \mapsto\left(T_{n} x, T_{n} y\right) \in Y^{2} \quad(n \in \mathbb{N})
$$

is hypercyclic. The sequence $\left(T_{n}\right)$ is called mixing if given a pair $U, V$ of nonempty open subsets of $X$, there is an $N \in \mathbb{N}$ such that $T_{n}(U) \cap V \neq \emptyset$ for every $n \geq N$. If $\left(T_{n}\right)$ is mixing, then it is weakly mixing, which in turn implies that $\left(T_{n}\right)$ is densely hypercyclic. For sharper relations among these three concepts, see [23], [11], [26], [15], [7], [5], [35], [4] and [25].

Now, we present a property that is a kind of mixing property depending only on the "shape" of the neighborhood. The concept is suggested by the notion of "Runge-transitive operators" introduced by Bonilla and GrosseErdmann in [16]. Observe that if $X, Y$ are two Fréchet spaces and $\left(T_{n}\right) \subset$ $L(X, Y)$, then $\left(T_{n}\right)$ is mixing if and only if, given $p \in \mathrm{SN}(X), q \in \mathrm{SN}(Y)$, a $p$-open set $U$ in $X$ and a $q$-open set $V$ in $Y$, there is $N=N(U, V) \in \mathbb{N}$ such that $T_{n}(U) \cap V \neq \emptyset$ for every $n \geq N$. Compare this with the definition below, which introduces a concept stronger than mixing.

Definition 3.2. If $X$ and $Y$ are Fréchet spaces and $\left(T_{n}\right) \subset L(X, Y)$, then we say that $\left(T_{n}\right)$ is supermixing if, given $p \in \mathrm{SN}(X)$ and $q \in \mathrm{SN}(Y)$, there exists $N=N(p, q) \in \mathbb{N}$ such that $T_{n}(U) \cap V \neq \emptyset$ for every $p$-open set $U$ in $X$, every $q$-open set $V$ in $Y$ and every $n \geq N$. An operator $T \in$ $L(X)$ is said to be supermixing whenever the sequence $\left(T^{n}\right)$ of its iterates is supermixing.

EXAMPles 3.3. 1. If $X$ is a Banach space, then no sequence $\left(T_{n}\right) \subset$ $L(X)$ can be supermixing. Indeed, supermixing in the Banach space case means that, for some $N$, the set $T_{N}(U) \cap V$ is nonempty for every pair $U, V$ of nonempty open sets, which is clearly impossible. 
2. Each translation operator $\tau_{a}(a \in \mathbb{C} \backslash\{0\})$ is supermixing on $\mathcal{E}$. Indeed, let $\left(p_{k}\right)$ be the sequence of seminorms $p_{k}(z)=\sup \{|f(z)|:|z| \leq k\}$ and fix $p, q \in \mathrm{SN}(\mathcal{E})$. Then there exist $k \in \mathbb{N}$ and $\delta>0$ such that $\{f: p(f)<1\}$ $\cap\{f: q(f)<1\} \supset\left\{f: p_{k}(f)<\delta\right\}$. Choose $N>2 k /|a|$, and fix an $n \geq N$, a $p$-open set $U$ and a $q$-open set $V$. Then there are $\alpha, \beta \in \mathcal{E}$ and $\varepsilon>0$ with $U \supset$ $\left\{f: p_{k}(f-\alpha)<\varepsilon\right\}$ and $V \supset\left\{f: p_{k}(f-\beta)<\varepsilon\right\}$. By Runge's approximation theorem applied to $\alpha, \beta$ on the compact set $\bar{B}(0, k) \cup \bar{B}(n a, k)$, there exists a polynomial $P$ such that $|P(z)-\alpha(z)|<\varepsilon$ on $\bar{B}(0, k)$ and $|P(z)-\beta(z-n a)|$ $<\varepsilon$ on $\bar{B}(n a, k)$. Then $p_{k}(P-\alpha)<\varepsilon$ and $|P(z+n a)-\beta(z)|<\varepsilon$ on $\bar{B}(0, k)$, so $p_{k}\left(\tau_{a}^{n} P-\beta\right)<\varepsilon$. Hence $P \in U \cap\left(\tau_{a}^{n}\right)^{-1}(V)$, which finishes the proof. This example will be generalized later (see Section 5 ).

3 . It is easy to check that the backward shift operator $B\left(x_{k}\right)=\left(x_{k+1}\right)$ on the Fréchet space $\omega=\mathbb{K}^{\mathbb{N}}$ of all scalar sequences (endowed with the product topology) is supermixing. Just use the denseness of the sequences having only finitely many nonzero entries, and take into account that, for every $p \in \mathrm{SN}(\omega)$, there are $k \in \mathbb{N}$ and $\varepsilon>0$ such that $\left\{x=\left(x_{j}\right) \in \omega: p(x)<1\right\}$ $\supset\left\{x \in \omega: \sup _{j \leq k}\left|x_{j}\right|<\varepsilon\right\}$.

4. Two criteria for disjoint hypercyclicity. We are now ready to state the first of our main results.

Theorem 4.1. Let $X, Y$ and $Z$ be three separable Fréchet spaces. Assume that $\left(S_{n}\right) \subset L(X, Y)$ and $\left(T_{n}\right) \subset L(X, Z)$. Suppose that the following conditions are satisfied:

(a) $\left(S_{n}\right)$ is densely hypercyclic.

(b) $\left(S_{n}\right)$ is $S N$-controlled.

(c) $\left(T_{n}\right)$ is supermixing.

Then $\left(S_{n}\right)$ and $\left(T_{n}\right)$ are densely disjoint hypercyclic.

Proof. We will use Remark 2.1.2. Fix nonempty open subsets $U, V, W$ of $X, Y, Z$, respectively. Then there are seminorms $p \in \mathrm{SN}(X), q \in \mathrm{SN}(Y)$, $r \in \mathrm{SN}(Z)$ as well as a $p$-open set $U_{0}$, a $q$-open set $V_{0}$ and an $r$-open set $W_{0}$ satisfying $U \supset U_{0}, V \supset V_{0}, W \supset W_{0}$. Since $\left(S_{n}\right)$ is SN-controlled, there is $\widetilde{p} \in \mathrm{SN}(X)$ such that $S_{n}^{-1}\left(V_{0}\right)$ is a $\widetilde{p}$-open set for all $n \in \mathbb{N}$. If $p_{0}:=\max \{p, \widetilde{p}\}$, then $p_{0} \in \mathrm{SN}(X)$ and each of the sets $U_{0}, S_{n}^{-1}\left(V_{0}\right)$ is $p_{0}$-open in $X$, hence so also is their intersection $U_{0} \cap S_{n}^{-1}\left(V_{0}\right)$, for every $n \in \mathbb{N}$. Since $\left(T_{n}\right)$ is supermixing, there is $N=N\left(p_{0}, r\right) \in \mathbb{N}$ such that $A \cap T_{n}^{-1}\left(W_{0}\right) \neq \emptyset$ for every $n \geq N$ and every nonempty $p_{0}$-open set $A$ in $X$. Finally, by the dense hypercyclicity of $\left(T_{n}\right)$, there is $n_{0} \geq N$ such that $U_{0} \cap S_{n_{0}}^{-1}\left(V_{0}\right) \neq \emptyset$. Hence we can take $A:=U_{0} \cap S_{n_{0}}^{-1}\left(V_{0}\right)$. It follows that $U_{0} \cap S_{n_{0}}^{-1}\left(V_{0}\right) \cap T_{n_{0}}^{-1}\left(W_{0}\right) \neq \emptyset$. Consequently, $U \cap S_{n_{0}}^{-1}(V) \cap T_{n_{0}}^{-1}(W) \neq \emptyset$, as required. 
REMARK 4.2. As a consequence, if $X$ is a Fréchet space and $S, T \in L(X)$ are such that $S$ is hypercyclic, the sequence $\left(S^{n}\right)$ is $\mathrm{SN}$-controlled and $T$ is supermixing, then $S$ and $T$ are densely disjoint hypercyclic.

The second criterion has to do with the Godefroy-Shapiro idea that an operator can be hypercyclic if it possesses a good supply of eigenvectors. This is the core of the proof of their theorem that an operator $\Phi(D)$ is hypercyclic (see [23, Section 5] and Section 5 below). Their idea was developed and formalized by the author in [6] to obtain hypercyclic sequences of differential operators. Now we extend Theorem 7 of [6] (see also [9]) to obtain a sufficient condition for disjoint hypercyclicity. Recall that, in a topological vector space, a subset is said to be total whenever its linear span is dense. If $T$ is an operator and $e$ is an eigenvector, then we denote by $\lambda(T, e)$ its corresponding eigenvalue.

TheOREM 4.3. Let $T_{1}, \ldots, T_{p}$ be operators on a separable $F$-space $X$. Assume that there are $p+1$ total subsets $D_{0}, D_{1}, \ldots, D_{p}$ of $X$ satisfying the following properties for all $j \in\{1, \ldots, p\}$ :

(a) Each vector $e \in D_{0} \cup D_{1} \cup \cdots \cup D_{p}$ is an eigenvector of $T_{j}$.

(b) $\left|\lambda\left(T_{j}, e\right)\right|<1$ for all $e \in D_{0}$.

(c) $\left|\lambda\left(T_{j}, e\right)\right|>1$ for all $e \in D_{j}$.

(d) $\left|\lambda\left(T_{j}, e\right)\right|<\left|\lambda\left(T_{i}, e\right)\right|$ for all $e \in D_{i}$ and all $i \in\{1, \ldots, p\} \backslash\{j\}$.

Then the sequence $\left(\left[T_{1}^{n}, \ldots, T_{p}^{n}\right]\right)$ is mixing. In particular, the operators $T_{1}, \ldots, T_{p}$ are densely disjoint hypercyclic.

Proof. First, note that $\lambda\left(S^{n}, e\right)=(\lambda(S, e))^{n}(n \in \mathbb{N})$ whenever $e$ is an eigenvector of the operator $S$. If $A \subset X$ denote by $s(A)$ the linear span of $A$. In view of the Birkhoff transitivity theorem-applied to the sequence $T_{n}=\left[T_{1}^{n}, \ldots, T_{p}^{n}\right]: X \rightarrow X^{p}(n \in \mathbb{N})$-it should be proved that, given nonempty open subsets $U, V_{1}, \ldots, V_{p}$ in $X$, there is $N \in \mathbb{N}$ such that $T_{j}^{n}(U) \cap V_{j} \neq \emptyset$ for all $j=1, \ldots, p$ and all $n \geq N$. Due to the denseness of $s\left(D_{0}\right), s\left(D_{1}\right), \ldots, s\left(D_{p}\right)$ in $X$, it is enough to prove that, given $p+1$ vectors $u_{j} \in s\left(D_{j}\right)(j=0, \ldots, p)$, there is a sequence $\left(x_{n}\right) \subset X$ such that, as $n \rightarrow \infty$,

$$
x_{n} \rightarrow u_{0} \quad \text { and } \quad T_{j}^{n} x_{n} \rightarrow u_{j} \quad(j=1, \ldots, p) .
$$

For fixed vectors $u_{j}(j=0,1, \ldots, p)$ as above, there are respective finite sets $F_{j}=\left\{e_{j, 1}, \ldots, e_{j, m(j)}\right\} \subset D_{j}$ and scalars $c_{j, 1}, \ldots, c_{j, m(j)}$ such that

$$
u_{j}=\sum_{l=1}^{m(j)} c_{j, l} e_{j, l} .
$$


By hypotheses (a)-(c), for every $j \in\{1, \ldots, p\}$,

$$
\begin{array}{ll}
\text { (2) } \quad \lambda\left(T_{j}^{n}, e_{0, l}\right)=\left(\lambda\left(T_{j}, e_{0, l}\right)\right)^{n} \rightarrow 0 \quad \text { as } n \rightarrow \infty(l=1, \ldots, m(0)), \\
\text { (3) } \lambda\left(T_{j}^{n}, e_{j, l}\right)=\left(\lambda\left(T_{j}, e_{j, l}\right)\right)^{n} \rightarrow \infty \quad \text { as } n \rightarrow \infty(l=1, \ldots, m(j)) .
\end{array}
$$

From condition (d) we obtain, for $i, j \in\{1, \ldots, p\}$ with $i \neq j$,

$$
\frac{\lambda\left(T_{j}^{n}, e_{i, l}\right)}{\lambda\left(T_{i}^{n}, e_{i, l}\right)}=\left(\frac{\lambda\left(T_{j}, e_{i, l}\right)}{\lambda\left(T_{i}, e_{i, l}\right)}\right)^{n} \rightarrow 0 \quad \text { as } n \rightarrow \infty(l=1, \ldots, m(i)) .
$$

Now, for each $n \in \mathbb{N}$, we define the vector

$$
x_{n}=u_{0}+\sum_{i=1}^{p} \sum_{l=1}^{m(i)} \frac{c_{i, l}}{\lambda\left(T_{i}^{n}, e_{i, l}\right)} e_{i, l} .
$$

By $(3), c_{i, l} / \lambda\left(T_{i}^{n}, e_{i, l}\right) \rightarrow 0(n \rightarrow \infty)$ for every $i=1, \ldots, p$ and every $l=1, \ldots, m(i)$, so $x_{n} \rightarrow u_{0}$. The first part of (1) has been proved. Finally, for each $j=1, \ldots, p$, we deduce from (2) and (4) that, as $n \rightarrow \infty$,

$$
\begin{aligned}
T_{j}^{n} x_{n}= & T_{j}^{n}\left(u_{0}+\sum_{i=1}^{p} \sum_{l=1}^{m(i)} \frac{c_{i, l}}{\lambda\left(T_{i}^{n}, e_{i, l}\right)} e_{i, l}\right) \\
= & \sum_{l=1}^{m(0)} c_{0, l} T_{j}^{n} e_{0, l}+\sum_{i=1}^{p} \sum_{l=1}^{m(i)} \frac{c_{i, l}}{\lambda\left(T_{i}^{n}, e_{i, l}\right)} T_{j}^{n} e_{i, l} \\
= & \sum_{l=1}^{m(0)} c_{0, l} \lambda\left(T_{j}^{n}, e_{0, l}\right) e_{0, l}+\sum_{i=1}^{p} \sum_{l=1}^{m(i)} \frac{c_{i, l}}{\lambda\left(T_{i}^{n}, e_{i, l}\right)} \lambda\left(T_{j}^{n}, e_{i, l}\right) e_{i, l} \\
= & \sum_{l=1}^{m(0)} c_{0, l} \lambda\left(T_{j}^{n}, e_{0, l}\right) e_{0, l}+\sum_{l=1}^{m(j)} c_{j, l} e_{j, l} \\
& +\sum_{i \in\{1, \ldots, p\} \backslash\{j\}}^{m(i)} \sum_{l=1}^{m(j)} \frac{c_{i, l}}{\lambda\left(T_{i}^{n}, e_{i, l}\right)} \lambda\left(T_{j}^{n}, e_{i, l}\right) e_{i, l} \\
\rightarrow & 0+\sum_{l=1}^{m} c_{j, l} e_{j, l}+0=u_{j} .
\end{aligned}
$$

This is the second part of (1), concluding the proof.

As in [6] and [9], there is a generalization of the last theorem to the case of sequences of operators $\left(T_{1, n}\right), \ldots,\left(T_{p, n}\right)$. This is left as an exercise for the interested reader.

5. Examples of disjoint hypercyclic operators. In this section, we furnish a number of examples of disjoint hypercyclic operators or sequences of operators. This will be done either directly or by applying Theorems 4.1 
and 4.3. We will consider composition operators and differential operators on spaces of analytic functions.

We recall that by Birkhoff's theorem [13] and MacLane's theorem [33], respectively, each nontrivial translation operator $\tau_{a}$ and the derivative operator $D$ are hypercyclic on $\mathcal{E}$. Moreover, as a link between $D$ and $\tau_{a}$, Godefroy and Shapiro [23] proved that $\Phi(D)$ is hypercyclic on $\mathcal{E}$ for any nonconstant entire function $\Phi \in$ Exp.

Let us start with a simple result concerning composition operators. If $G \subset \mathbb{C}$ is a domain and $\varphi: G \rightarrow G$ is a holomorphic selfmap, then the composition operator $C_{\varphi} \in L(H(G))$ generated by $\varphi$ is defined as $C_{\varphi} f=$ $f \circ \varphi$. We denote by $\operatorname{Aut}(G)$ the group of its automorphisms, that is, of its bijective holomorphic selfmaps. Recall that a domain $G$ is called simply connected whenever $\mathbb{C}_{\infty} \backslash G$ is connected, where $\mathbb{C}_{\infty}=\mathbb{C} \cup\{\infty\}$ is the extended plane.

TheOREM 5.1. Assume that $G$ is a simply connected domain, $m \in \mathbb{N}$, and $\left(\varphi_{j, n}\right) \subset \operatorname{Aut}(G)(j=1, \ldots, m)$. Suppose that these sequences "leave compacta separately", that is, for every compact subset $K \subset G$, there is $N=$ $N(K) \in \mathbb{N}$ such that, for each $n \geq N$, the sets $K, \varphi_{1, n}(K), \ldots, \varphi_{m, n}(K)$ are pairwise disjoint. Then the sequence $\left[C_{\varphi_{1, n}}, \ldots, C_{\varphi_{m, n}}\right]: H(G) \rightarrow(H(G))^{m}$ $(n \in \mathbb{N})$ is supermixing. In particular, the sequences $\left(C_{\varphi_{1, n}}\right), \ldots,\left(C_{\varphi_{m, n}}\right)$ are densely disjoint hypercyclic.

Proof. Let $p \in \mathrm{SN}(H(G))$ and $q \in \mathrm{SN}\left((H(G))^{m}\right)$. Since $G$ is simply connected, one can find a compact set $K \subset G$ without holes and a $\delta>0$ such that $\{f: p(f)<1\} \supset\left\{f: \sup _{K}|f|<\delta\right\}$ and $\left\{F=\left(f_{1}, \ldots, f_{m}\right)\right.$ : $q(F)<1\} \supset\left\{F: \sup _{K}\left|f_{j}\right|<\delta(j=1, \ldots, m)\right\}$. Let $N=N(K)$ be the natural number furnished by the "leaving" hypothesis. Fix $n \geq N$. Fix also a nonempty $p$-open set $U \subset H(G)$ and a nonempty $q$-open set $V \subset(H(G))^{m}$. Then we derive the existence of functions $\alpha, \beta_{1}, \ldots, \beta_{m} \in H(G)$ and of a number $\varepsilon>0$ such that $U \supset\{f \in H(G):|f(z)-\alpha(z)|<\varepsilon$ for all $z \in K\}$ and $V \supset\left\{F \in(H(G))^{m}:\left|f_{j}(z)-\beta_{j}(z)\right|<\varepsilon\right.$ for all $z \in K$ and all $j=1, \ldots, m\}$. Since each $\varphi_{j, n}$ is an automorphism, the compact set $\varphi_{j, n}(K)$ has no holes. Hence the disjoint union $K \cup \varphi_{1, n}(K) \cup \cdots \cup$ $\varphi_{m, n}(K)$ is a compact set without holes. Therefore, Runge's theorem yields a polynomial $f$ (so $f \in H(G)$ ) such that $|f(z)-\alpha(z)|<\varepsilon$ on $K$ and $\left|f(z)-\beta_{j}\left(\varphi_{j, n}^{-1}(z)\right)\right|<\varepsilon$ on $\varphi_{j, n}(K)(j=1, \ldots, m)$. The last set of inequalities is equivalent to $\left|\left(C_{\varphi_{j, n}} f\right)(z)-\beta_{j}(z)\right|<\varepsilon$ on $K(j=1, \ldots, m)$. Therefore $f \in U$ and $\left[C_{\varphi_{1, n}}, \ldots, C_{\varphi_{m, n}}\right] f \in V$. Hence $\left[C_{\varphi_{1, n}}, \ldots, C_{\varphi_{m, n}}\right](U) \cap V \neq \emptyset$, as required.

REMARKs 5.2. 1. The "leaving" condition of Theorem 5.1 is fulfilled if the sequences $\left(\varphi_{j, n}\right)$ tend uniformly on compacta in $G$-in the sense of the 
chordal distance - to $m$ distinct points of $\partial G$, the boundary of $G$ in $\mathbb{C}_{\infty}$. This is not at all necessary (see Proposition 5.5).

2. In the case $m=1$, the supermixing conclusion of Theorem 5.1 still holds just on assuming that $G$ is a domain of $\mathbb{C}$ which is not isomorphic to the punctured plane $\mathbb{C} \backslash\{0\}$. That is, if $\left(\varphi_{n}\right) \subset \operatorname{Aut}(G)$ and $\varphi_{n} \rightarrow \partial G$ compactly, then the sequence $\left(C_{\varphi_{n}}\right)$ is supermixing. Indeed, by adapting the proof of Lemma 2.12 in [8] from the so-called "run-away" sequences of automorphisms to our $\left(\varphi_{n}\right)$ (the lemma dealt with domains of infinite connectivity; its conclusion is evident for simply connected domains, while for domains with finite connectivity $\geq 3$ or isomorphic to an annulus or to $\mathbb{D} \backslash\{0\}$, its conclusion is trivial because there is no sequence of automorphisms tending to $\partial G$; in the case of finite connectivity $\geq 3$, this is so by Heins' theorem [29]), we find that if $K$ is a compact subset of $G$ such that each hole of $K$ contains some hole of $G$, then there is $N=N(K) \in \mathbb{N}$ such that, for all $n \geq N$, we have $K \cap \varphi_{n}(K)=\emptyset$ and $K \cup \varphi_{n}(K)$ is a compact set each of whose holes contains at least one hole of $G$. This yields, via Runge's theorem and similarly to the final part of the proof of Theorem 5.1, a rational function $f$ with poles outside $G$ (so $f \in H(G)$ ) such that $|f(z)-\alpha(z)|<\varepsilon$ on $K$ and $\left|f(z)-\beta\left(\varphi_{n}^{-1}(z)\right)\right|<\varepsilon$ on $\varphi_{n}(K)$, for any preassigned $\varepsilon>0$ and $\alpha, \beta \in H(G)$. This completes the proof.

Next, we turn our attention to differential operators. In the following statement, which concerns operators $\Phi(D)$ on $H(G)$, we may suppose merely that $\Phi \in \operatorname{Exp}$ if $G$ is the whole plane $\mathbb{C}$. In addition, the statement has a multi-dimensional version - with the same proof except for small changes of notation - if the expression "simply connected domain" is replaced by "Runge domain" (i.e., a domain in $\mathbb{C}^{N}$ such that the set of polynomials is dense in $H(G)$; see [31] or [32, Chap. 5] for properties and characterizations). In the definition of entire function of exponential or subexponential type, one should interpret the modulus $|z|$ of a point $z=\left(z_{1}, \ldots, z_{N}\right)$ as its euclidean norm $\left(\left|z_{1}\right|^{2}+\cdots+\left|z_{N}\right|^{2}\right)^{1 / 2}$.

TheOREM 5.3. Assume that $G$ is a simply connected domain of $\mathbb{C}$ and that $\Phi_{1}, \ldots, \Phi_{p}$ are entire functions of subexponential type. Assume also that there are $p+1$ points $z_{0}, z_{1}, \ldots, z_{p} \in \mathbb{C}$ such that:

(a) $\max \left\{\left|\Phi_{j}\left(z_{0}\right)\right|: j=1, \ldots, p\right\}<1$.

(b) $\max \left\{1,\left|\Phi_{j}\left(z_{i}\right)\right|: j \in\{1, \ldots, p\} \backslash\{i\}\right\}<\left|\Phi_{i}\left(z_{i}\right)\right|$ for all $i=1, \ldots, p$.

Then the operators $\Phi_{1}(D), \ldots, \Phi_{p}(D)$ are densely disjoint hypercyclic on $H(G)$.

Proof. For $a \in \mathbb{C}$ and $A \subset \mathbb{C}$, set $e_{a}(z):=\exp (a z)$ and $M(A):=\left\{e_{a}:\right.$ $a \in A\}$. If $A$ is a nonempty open set, then $M(A)$ is total in $H(G)$ (see for instance [23, Section 5] or [9, Lemma 2.3]). Observe that $\Phi(D) e_{a}=$ 
$\Phi(a) e_{a}$ for all $a \in \mathbb{C}$ and all $\Phi \in \operatorname{Exp}$, so $e_{a}$ is an eigenvector of $\Phi(D)$ with eigenvalue $\Phi(a)$. By the continuity of the functions $\left|\Phi_{j}\right|$, there are nonempty open subsets $U_{j} \subset \mathbb{C}$ such that, for every $j \in\{1, \ldots, p\}$, one has $\left|\Phi_{j}(z)\right|<1$ on $U_{0},\left|\Phi_{j}(z)\right|>1$ on $U_{j}$, and $\left|\Phi_{i}(z)\right|>\left|\Phi_{j}(z)\right|$ on $U_{i}$ whenever $i \neq j$. Thus, it suffices to apply Theorem 4.3 to $T_{j}:=\Phi_{j}(D)$ and $D_{j}:=M\left(U_{j}\right)$.

ExAmPles 5.4. 1. Approximation by Birkhoff-universal functions and MacLane-universal functions can be simultaneously performed with the same functions and with the same approximating iterates. In other words, the operators $\tau_{1}$ and $D$ are disjoint hypercyclic on $\mathcal{E}$. Indeed, $\tau_{1}=\Phi_{1}(D)$ and $D=\Phi_{2}(D)$, where $\Phi_{1}(z):=e^{z}$ and $\Phi_{2}(z):=z$. Then just apply Theorem 5.3 with $p=2, z_{0}=-1 / 2, z_{1}=1$ and $z_{2}=-1$. This result can also be derived from Example 3.2.1, Theorem 4.1 and Remark 4.2, because $\tau_{1}$ is supermixing, $D$ is hypercyclic and $\left(D^{n}\right)$ is SN-controlled.

2. Of course, one can mix Theorems 5.1 and 5.3 to obtain (with the help of Theorem 4.1) more examples of disjoint hypercyclic operators.

The final part of this section is devoted to several examples (in the cases $G=\mathbb{C}$ or $\mathbb{D}$ ) of disjoint hypercyclic sequences of composition operators, via an application of Theorem 5.1.

Recall that the members of Aut($(\mathbb{C})$ are the similarities $z \mapsto a z+b$ $(a, b \in \mathbb{C}, a \neq 0)$, while Aut $(\mathbb{D})$ consists of the Möbius transformations of the form $z \mapsto k \frac{z-a}{1-\bar{a} z}(|a|<1=|k|)$. As a generalization of Birkhoff's theorem and of Seidel-Walsh's theorem (the latter is the $\mathbb{D}$-version of the Birkhoff theorem, see [39]), it is known that if $\left(\varphi_{n}(z):=a_{n} z+b_{n}\right) \in \operatorname{Aut}(\mathbb{C})$ $\left(\left(\varphi_{n}(z):=k_{n} \frac{z-a_{n}}{1-\bar{a}_{n} z}\right) \in \operatorname{Aut}(\mathbb{D})\right.$, resp.) then $\left(C_{\varphi_{n}}\right)$ is hypercyclic on $\mathcal{E}$ (on $H(\mathbb{D})$, resp.) if and only if $\sup _{n \in \mathbb{N}} \min \left\{\left|b_{n}\right|,\left|b_{n} / a_{n}\right|\right\}=+\infty\left(\sup _{n \in \mathbb{N}}\left|a_{n}\right|=1\right.$, resp.). Moreover, if $\varphi(z):=a z+b \in \operatorname{Aut}(\mathbb{C})$, then $C_{\varphi}$ is hypercyclic on $\mathcal{E}$ if and only if $\varphi$ is a translation, that is, $a=1$ and $b \neq 0$. And if $\varphi(z):=e^{i \theta} \frac{z-a}{1-\bar{a} z} \in \operatorname{Aut}(\mathbb{D})$, then $C_{\varphi}$ is hypercyclic on $H(\mathbb{D})$ if and only if $\varphi$ has no fixed point in $\mathbb{D}$, or equivalently, if and only if $|a| \geq|\sin (\theta / 2)|$ and $a \neq 0$ (for this and extensions, see [8], [18] and [40]). In particular, every "non-Euclidean translation" $\varphi(z):=z * a=\frac{z+a}{1+\bar{a} z}(a \in \mathbb{D} \backslash\{0\})$ generates a hypercyclic composition operator on $H(\mathbb{D})$.

Proposition 5.5.

(A) If $b_{1}, \ldots, b_{p}$ are pairwise different nonzero complex numbers, then the translation operators $\tau_{b_{1}}, \ldots, \tau_{b_{p}}$ are densely disjoint hypercyclic on the space $\mathcal{E}$.

(B) Assume that $\varphi_{j, n}(z):=a_{j, n} z+b_{j, n}(j=1, \ldots, p ; n \in \mathbb{N})$ are automorphisms of $\mathbb{C}$ satisfying, for every $j \in\{1, \ldots, p\}$, the following properties: 

(a) $\lim _{n \rightarrow \infty}\left|b_{j, n}\right|=\infty=\lim _{n \rightarrow \infty}\left|\frac{b_{j, n}}{a_{j, n}}\right|$.
(b) $\lim _{n \rightarrow \infty} \frac{\left|b_{i, n}-b_{j, n}\right|}{\left|a_{i, n}\right|+\left|a_{j, n}\right|}=\infty$ for every $i \in\{1, \ldots, p\} \backslash\{j\}$.

Then the sequence $\left[C_{\varphi_{1, n}}, \ldots, C_{\varphi_{p, n}}\right]: \mathcal{E} \rightarrow \mathcal{E}^{p}(n \in \mathbb{N})$ is supermixing. In particular, the sequences $\left(C_{\varphi_{1, n}}\right), \ldots,\left(C_{\varphi_{p, n}}\right)$ are densely disjoint hypercyclic on $\mathcal{E}$.

Proof. Observe that (A) follows from (B) applied to $a_{j, n}:=1, b_{j, n}:=$ $n b_{j}$. Therefore, it suffices to demonstrate (B). For this, fix a compact set $K \subset \mathbb{C}$. Choose $r>0$ such that $K \subset \bar{B}(0, r)=: B$. By hypothesis, there is a positive integer $N$ such that, for every $j \in\{1, \ldots, p\}$ and every $n \geq N$, we have $\left|b_{j, n}\right|>2 r,\left|b_{j, n} / a_{j, n}\right|>2 r$, and $\left|b_{i, n}-b_{j, n}\right|>\left|a_{i, n}\right| r+\left|a_{j, n}\right| r$ for all $i \in\{1, \ldots, p\} \backslash\{j\}$. Then, if $n \geq N$, the $p$ balls $\bar{B}\left(b_{j, n},\left|a_{j, n}\right| r\right)=\varphi_{j, n}(B)$ are pairwise disjoint. Furthermore, we have

$$
\left|\varphi_{j, n}(z)\right|=\left|b_{j, n}\right|\left|1+\frac{a_{j, n} z}{b_{j, n}}\right| \geq\left|b_{j, n}\right|\left(1-\left|\frac{a_{j, n} z}{b_{j, n}}\right|\right)>2 r\left(1-\frac{r}{2 r}\right)=r
$$

for all $z \in B$, all $j \in\{1, \ldots, p\}$ and all $n \geq N$. Then $B \cap \varphi_{n, j}(B)=\emptyset$ for all $j$ and all $n \geq N$. Since $K \subset B$, we infer that, for each $n \geq N$, the sets $K, \varphi_{1, n}(K), \ldots, \varphi_{p, n}(K)$ are pairwise disjoint. The conclusion follows from Theorem 5.1.

If $a \in \mathbb{C}$, then we denote by $a^{*}$ the point symmetric to $a$ with respect to the unit circle $\partial \mathbb{D}$, that is, $a^{*}=1 / \bar{a}$.

Proposition 5.6.

(A) If $a_{1}, \ldots, a_{p}$ are pairwise different points in $\mathbb{D}$ and $\varphi_{j}(z):=z * a_{j}$ $(j=1, \ldots, p)$ are their respective non-Euclidean translations, then the composition operators $C_{\varphi_{1}}, \ldots, C_{\varphi_{p}}$ are densely disjoint hypercyclic.

(B) Suppose that $\left\{\varphi_{j, n}: j \in\{1, \ldots, p\}, n \in \mathbb{N}\right\} \subset \operatorname{Aut}(\mathbb{D})$. Assume also that, for each $j \in\{1, \ldots, p\}$, the following properties are satisfied:

(a) $\lim _{n \rightarrow \infty}\left|\varphi_{j, n}(0)\right|=1$.

(b) $\lim _{n \rightarrow \infty}\left|\frac{\varphi_{i, n}(0)-\varphi_{j, n}(0)}{\varphi_{i, n}(0)-\left(\varphi_{j, n}(0)\right)^{*}}\right|=1$ for all $i \in\{1, \ldots, p\} \backslash\{j\}$.

Then the sequences $\left(C_{\varphi_{1, n}}\right), \ldots,\left(C_{\varphi_{p, n}}\right)$ are densely disjoint hypercyclic.

Proof. Assuming that (B) is proved, let us demonstrate (A). Let $\varphi$ be a non-Euclidean translation $\varphi(z)=z * a$ with $a=r e^{i \theta}$, where $r \in(0,1)$ and $\theta \in[0,2 \pi)$. Define $\alpha$ as the simplest Möbius transformation sending $1,-1$ 
to $0, \infty$, respectively, and $\beta$ as the rotation of angle $\theta$, that is,

$$
\alpha(z)=\frac{z-1}{z+1} \quad \text { and } \quad \beta(z)=e^{i \theta} z .
$$

Then $\alpha^{-1}(z)=(1+z) /(1-z)$ and $\beta^{-1}(z)=e^{-i \theta} z$. If $\psi$ is defined as $\psi=$ $\alpha^{-1} \circ \beta^{-1} \circ \varphi \circ \beta \circ \alpha$, then an easy calculation shows that $\psi$ is the dilation $\psi=r_{0} z$, where $r_{0}=(1+r) /(1-r)(>1)$. Let $\varphi_{n}, \psi_{n}$ denote the $n$th iterate of $\varphi, \psi$, respectively. Since $\varphi=\beta \circ \alpha \circ \psi \circ \alpha^{-1} \circ \beta^{-1}$, one obtains $\varphi_{n}=\beta \circ \alpha \circ \psi_{n} \circ \alpha^{-1} \circ \beta^{-1}$. But $\psi_{n}(z)=r_{0}^{n} z$, whence

$$
\varphi_{n}(0)=\beta\left(\alpha\left(\psi_{n}(1)\right)\right)=e^{i \theta} \frac{r_{0}^{n}-1}{r_{0}^{n}+1} .
$$

Note that, as $n \rightarrow \infty$, we get $\varphi_{n}(0) \rightarrow e^{i \theta}$, so $\left|\varphi_{n}(0)\right| \rightarrow 1$.

Consequently, according to (B), it is enough to prove that, if $\varphi(z):=$ $z * a, \Phi(z):=z * b$ are two non-Euclidean translations (with $a=r e^{i \theta} \neq$ $R e^{i \Theta}=b, 0<r \leq R<1$ and $\left.\theta, \Theta \in[0,2 \pi)\right)$ and $\varphi_{n}, \Phi_{n}$ denote their respective iterates, then

$$
A_{n}:=\left|\frac{\varphi_{n}(0)-\Phi_{n}(0)}{\varphi_{n}(0)-\left(\Phi_{n}(0)\right)^{*}}\right| \rightarrow 1 \quad \text { as } n \rightarrow \infty .
$$

At this point, we distinguish two cases. If $\theta \neq \Theta$, then

$$
A_{n} \rightarrow\left|\frac{e^{i \theta}-e^{i \Theta}}{e^{i \theta}-\left(e^{i \Theta}\right)^{-1}}\right|=1 .
$$

Finally, if $\theta=\Theta$, then $r<R$. We set

$$
r_{0}:=\frac{1+r}{1-r} \quad \text { and } \quad R_{0}:=\frac{1+R}{1-R}
$$

so that $R_{0}>r_{0}>1$. Then

$$
A_{n}=\left|\frac{\frac{r_{0}^{n}-1}{r_{0}^{n}+1}-\frac{R_{0}^{n}-1}{R_{0}^{n}+1}}{\frac{r_{0}^{n}-1}{r_{0}^{n}+1}-\frac{R_{0}^{n}+1}{R_{0}^{n}-1}}\right|=\frac{R_{0}^{n}-1}{R_{0}^{n}+1} \frac{\left(R_{0} / r_{0}\right)^{n}-1}{\left(R_{0} / r_{0}\right)^{n}+1} \rightarrow 1 \cdot 1=1,
$$

and we are done.

Next, we prove (B). For each pair $(j, n)$, we can write

$$
\varphi_{j, n}(z)=k_{j, n} \frac{z-a_{j, n}}{1-\bar{a}_{j, n} z}
$$

for certain numbers $a_{j, n}, k_{j, n}$ satisfying $\left|a_{j, n}\right|<1=\left|k_{j, n}\right|$. Then $\varphi_{j, n}(0)=$ $-k_{j, n} a_{j, n}$ and $\left|\varphi_{j, n}(0)\right|=\left|a_{j, n}\right|$. Fix a compact set $K \subset \mathbb{D}$. Choose a ball $B=\bar{B}(0, r)$ with $r \in(0,1)$ and $K \subset B$. As $\left|\varphi_{j, n}(0)\right| \rightarrow 1(n \rightarrow \infty)$ for all $j$, we can find $N_{1} \in \mathbb{N}$ such that $\left|a_{j, n}\right|>\left(2 r-r^{2}\right)^{1 / 2}$ for all $j \in\{1, \ldots, p\}$ and 
all $n \geq N_{1}$. Therefore, if $z \in B$ and $j, n$ are as before, we get

$$
\begin{aligned}
\left|\varphi_{j, n}(z)\right| & =\left|\frac{z-a_{j, n}}{1-\bar{a}_{j, n} z}\right|=\frac{1}{\left|\bar{a}_{j, n}\right|}\left|\frac{\left|a_{j, n}\right|^{2}-\bar{a}_{j, n} z}{1-\bar{a}_{j, n} z}\right|=\frac{1}{\left|a_{j, n}\right|}\left|1+\frac{\left|a_{j, n}\right|^{2}-1}{1-\bar{a}_{j, n} z}\right| \\
& \geq 1-\frac{1-\left|a_{j, n}\right|^{2}}{\left|1-\bar{a}_{j, n} z\right|} \geq 1-\frac{1-\left|a_{j, n}\right|^{2}}{1-|z|} \geq 1-\frac{1+r^{2}-2 r}{1-r}=r .
\end{aligned}
$$

Hence $B \cap \varphi_{j, n}(B)=\emptyset$, so $K \cap \varphi_{j, n}(K)=\emptyset\left(n \geq N_{1}, j \in\{1, \ldots, p\}\right)$. According to Theorem 5.1, and since $K \subset B$, it is enough to show the existence of a positive integer $N \geq N_{1}$ such that $\varphi_{i, n}(B) \cap \varphi_{j, n}(B)=\emptyset$ for all $i, j$ with $i \neq j$ and all $n \geq N$.

For this, consider the hyperbolic distance $\varrho$ on $\mathbb{D}$ (see [21]), given by

$$
\varrho(z, w)=\tanh ^{-1}\left|\frac{z-w}{1-z \bar{w}}\right| \quad(z, w \in \mathbb{D}) .
$$

Since $\varrho$ is invariant under automorphisms, we deduce from the triangle inequality that, for $z, w \in B, n \in \mathbb{N}$ and $i, j \in\{1, \ldots, p\}$,

$$
\begin{aligned}
& \varrho\left(\varphi_{i, n}(z), \varphi_{j, n}(w)\right) \\
& \quad \geq \varrho\left(\varphi_{i, n}(0), \varphi_{j, n}(0)\right)-\varrho\left(\varphi_{i, n}(z), \varphi_{i, n}(0)\right)-\varrho\left(\varphi_{j, n}(w), \varphi_{j, n}(0)\right) \\
& \quad=\varrho\left(\varphi_{i, n}(0), \varphi_{j, n}(0)\right)-\varrho(z, 0)-\varrho(w, 0) \geq \varrho\left(\varphi_{i, n}(0), \varphi_{j, n}(0)\right)-2 \tanh ^{-1} r .
\end{aligned}
$$

Since (a) and (b) hold and $\lim _{x \rightarrow 1^{-}} \tanh ^{-1} x=\infty$, for every pair $i, j$ with $i \neq j$ we obtain

$$
\begin{aligned}
\lim _{n \rightarrow \infty} \varrho\left(\varphi_{i, n}(0), \varphi_{j, n}(0)\right) & =\lim _{n \rightarrow \infty} \tanh ^{-1}\left|\frac{\varphi_{i, n}(0)-\varphi_{j, n}(0)}{1-\varphi_{i, n}(0) \overline{\varphi_{j, n}(0)}}\right| \\
& =\lim _{n \rightarrow \infty} \tanh ^{-1}\left(\frac{1}{\left|\overline{\varphi_{j, n}(0)}\right|}\left|\frac{\varphi_{i, n}(0)-\varphi_{j, n}(0)}{\varphi_{i, n}(0)-\left(\varphi_{j, n}(0)\right)^{*}}\right|\right) \\
& =\lim _{n \rightarrow \infty} \tanh ^{-1}\left|\frac{\varphi_{i, n}(0)-\varphi_{j, n}(0)}{\varphi_{i, n}(0)-\left(\varphi_{j, n}(0)\right)^{*}}\right|=\infty .
\end{aligned}
$$

Hence $\lim _{n \rightarrow \infty} \varrho\left(\varphi_{i, n}(0), \varphi_{j, n}(0)\right)=\infty$, so there exist $N \in \mathbb{N}$ (we can choose $\left.N \geq N_{1}\right)$ such that $\varrho\left(\varphi_{i, n}(0), \varphi_{j, n}(0)\right)>2 \tanh ^{-1} r$ for all $n \geq N$ and all $i, j$ with $i \neq j$. Consequently, $\varrho\left(\varphi_{i, n}(z), \varphi_{j, n}(w)\right)>0$ for such $i, j, n$ and all $z, w \in B$. But this implies $\varphi_{i, n}(B) \cap \varphi_{j, n}(B)=\emptyset$, as required.

We finish this paper by providing an example of disjoint hypercyclic operators in the setting of Banach spaces. In 1969, S. Rolewicz [36] gave the first example of a hypercyclic operator on a Banach space. Namely, he proved that if $c \in \mathbb{K}$ has modulus $>1$ and $B$ is the backward shift on any of the spaces $l^{p}\left(=\right.$ the space of scalar sequences $x=\left(x_{j}\right)$ with $\left.\|x\|:=\left(\sum_{j=1}^{\infty}\left|x_{j}\right|^{p}\right)<\infty\right)(1 \leq p<\infty)$ or $c_{0}(=$ the space of scalar sequences $x=\left(x_{j}\right)$ tending to 0 , endowed with the norm $\left.\|x\|:=\sup _{j \geq 1}\left|x_{j}\right|\right)$, then the operator $c B$ is hypercyclic. In 1995, H. Salas [38] improved this 
result by showing that, if $a=\left(a_{j}\right)$ is a bounded sequence of nonzero scalars, then the weighted backward shift operator $T_{a}$ defined on $l^{p}$ or $c_{0}$ as

$$
T_{a} x=\left(a_{j+1} x_{j+1}\right) \quad \text { for } x=\left(x_{j}\right)
$$

is hypercyclic if and only if the sequence $\left\{A_{n}:=\prod_{j=1}^{n} a_{j}\right\}_{n \geq 1}$ of partial products of the weights is unbounded. Extensions of Salas' result can be found for example in [11], [27] and [34]. Since every power of a hypercyclic operator is also hypercyclic (even with the same hypercyclic vectors, see [1]), it follows that, if $a$ is as above, then $T_{a}^{m}$ is hypercyclic for every $m \in \mathbb{N}$. In fact, we have the following statement.

Proposition 5.7. Let $X$ be $c_{0}$ or $l^{p}(1 \leq p<\infty)$, and let $m \in \mathbb{N}$. Assume that $a=\left(a_{j}\right)$ is a bounded sequence of nonzero scalars such that

$$
\sup \left\{\min \left\{\left|A_{n}\right|,\left|A_{2 n}\right|, \ldots,\left|A_{m n}\right|\right\}\right\}=\infty .
$$

Then the operators $T_{a}, T_{a}^{2}, \ldots, T_{a}^{m}$ are densely disjoint hypercyclic on $X$.

Proof. The proof will be given when $m=2$ and $X=l^{p}$, the general case being similar. If $T:=T_{a}$, then it must be proved that the sequence $\left(\left[T^{n}, T^{2 n}\right]\right)$ is densely disjoint hypercyclic. From the Birkhoff transitivity theorem it is enough to find dense subsets $D_{0}, D_{1}, D_{2}$ of $X$ such that, for $x \in D_{0}, y \in D_{1}$ and $z \in D_{2}$, there are a sequence $\left\{n_{1}<n_{2}<\cdots\right\} \subset \mathbb{N}$ and a sequence $\left(x_{k}\right) \subset X$ satisfying:

(i) $T^{n_{k}} x \rightarrow 0$ and $T^{2 n_{k}} x \rightarrow 0$ as $k \rightarrow \infty$.

(ii) $x_{k} \rightarrow 0, T^{n_{k}} x_{k} \rightarrow y$ and $T^{2 n_{k}} x_{k} \rightarrow z$ as $k \rightarrow \infty$.

We choose $D_{0}=D_{1}=D_{2}:=D$, where $D$ is the set of sequences with finitely many nonzero entries. Given $x \in D$, there is $n_{0} \in \mathbb{N}$ such that $T^{n} x=0$ for every $n \geq n_{0}$, so condition (i) is trivially satisfied for any $\left(n_{k}\right)$. Now, fix $y=\left(y_{1}, \ldots, y_{q}, 0,0,0, \ldots\right)$ and $z=\left(z_{1}, \ldots, z_{r}, 0,0,0, \ldots\right)$ in $D$. Without loss of generality, we may suppose that $q=r$. By (5), there is a sequence $\left\{m_{1}<m_{2}<\cdots\right\} \subset \mathbb{N}$ such that $A_{m_{k}} \rightarrow \infty$ and $A_{2 m_{k}} \rightarrow \infty$. We can assume that $m_{1}>2 q$ (so $m_{k}-q>q$ for all $k$ ). For $k \in \mathbb{N}$, define $n_{k} \in \mathbb{N}$ and $x_{k} \in X$ by $n_{k}:=m_{k}-q$ and

$$
\begin{aligned}
x_{k}:= & \left(0, \ldots, 0, A_{1} A_{n_{k}+1}^{-1} y_{1}, A_{2} A_{n_{k}+2}^{-1} y_{2}, \ldots, A_{q} A_{n_{k}+q}^{-1} y_{q}, 0, \ldots, 0,\right. \\
& \left.A_{1} A_{n_{2 k}+1}^{-1} z_{1}, A_{2} A_{n_{2 k}+2}^{-1} z_{2}, \ldots, A_{q} A_{n_{2 k}+q}^{-1} z_{q}, 0,0,0, \ldots\right)
\end{aligned}
$$

where there are $n_{k}$ zeros as starting entries and $n_{k}-q$ zeros immediately after $A_{q} A_{n_{k}+q}^{-1} y_{q}$. Observe that, by construction, $T^{2 n_{k}} x_{k}=z \rightarrow z(k \rightarrow \infty)$. So part of (ii) has been shown. Hence, it remains to prove that $x_{k} \rightarrow 0$ and $T^{n_{k}} x_{k} \rightarrow y$. Fix $j \in\{1, \ldots, q\}$. By hypothesis, there is $M \in(0, \infty)$ such that $\left|a_{l}\right| \leq M$ for all $l$. Therefore

$$
\left|A_{j} A_{n_{k}+j}^{-1}\right| \leq M^{j}\left|A_{m_{k}-q+j}^{-1}\right| \leq M^{j}\left|A_{m_{k}}^{-1}\right| M^{q-j}=M^{q}\left|A_{m_{k}}^{-1}\right|
$$


and, analogously, $\left|A_{j} A_{2 n_{k}+j}^{-1}\right| \leq M^{2 q}\left|A_{2 m_{k}}^{-1}\right|$. Consequently,

$$
\left\|x_{k}\right\|^{p} \leq\left(M^{q}\left|A_{m_{k}}^{-1}\right|\right)^{p} \sum_{j=1}^{q}\left|y_{j}\right|^{p}+\left(M^{2 q}\left|A_{2 m_{k}}^{-1}\right|\right)^{p} \sum_{j=1}^{q}\left|z_{j}\right|^{p} \rightarrow 0
$$

and

$$
\begin{aligned}
\left\|T^{n_{k}} x_{k}-y\right\|^{p} & =\left|\left(A_{1} A_{n_{k}+1}^{-1} z_{1}, \ldots, A_{q} A_{n_{k}+q}^{-1} z_{q}, 0,0,0, \ldots\right)\right|^{p} \\
& \leq\left(M^{q}\left|A_{m_{k}}^{-1}\right|\right)^{p} \sum_{j=1}^{q}\left|z_{j}\right|^{p} \rightarrow 0 .
\end{aligned}
$$

But this shows that $x_{k} \rightarrow 0$ and $T^{n_{k}} x_{k} \rightarrow y$, as required.

Final questions. 1. If $T$ is a hypercyclic operator, are $T, T^{2}$ always disjoint hypercyclic?

2. Is there any Banach space example where Theorem 4.3 can be applied?

3. Are finitely many composition operators generated by nonelliptic (= without fixed points in $\mathbb{D}$ ) automorphisms $\varphi$ of $\mathbb{D}$ disjoint hypercyclic? Is this true for the Hardy space $H^{p}(\mathbb{D})$, or even for other Banach spaces $X \subset H(\mathbb{D})$ where $C_{\varphi}$ is a well-defined operator?

Acknowledgements. We are grateful to the referee for helpful comments and suggestions, and to A. Peris for informing us, during the process of refereeing of this paper, that the concept introduced here has also been studied by him and J. P. Bès in [12]. We are also indebted to K. G. GrosseErdmann for providing us with a definition of SN-control of $\left(T_{n}\right)$ that is more natural than the one given in an earlier version.

\section{References}

[1] S. I. Ansari, Hypercyclic and cyclic vectors, J. Funct. Anal. 128 (1995), 374-383.

[2] F. Bayart, Topological and algebraic genericity of divergence and universality, Studia Math. 167 (2005), 161-181.

[3] C. A. Berenstein and R. Gay, Complex Analysis and Special Topics in Harmonic Analysis, Springer, New York, 1995.

[4] T. Bermúdez, A. Bonilla, J. A. Conejero and A. Peris, Hypercyclic, topologically mixing and chaotic semigroups on Banach spaces, Studia Math. 170 (2005), 57-75.

[5] T. Bermúdez, A. Bonilla and A. Peris, On hypercyclicity and supercyclicity criteria, Bull. Austral. Math. Soc. 70 (2004), 45-54.

[6] L. Bernal-González, Hypercyclic sequences of differential and antidifferential operators, J. Approx. Theory 96 (1999), 323-337.

[7] L. Bernal-González and K. G. Grosse-Erdmann, The Hypercyclicity Criterion for sequences of operators, Studia Math. 157 (2003), 17-32.

[8] L. Bernal-González and A. Montes-Rodríguez, Universal functions for composition operators, Complex Variables Theory Appl. 27 (1995), 47-56. 
[9] L. Bernal-González and J. A. Prado-Tendero, Sequences of differential operators: exponentials, hypercyclicity and equicontinuity, Ann. Polon. Math. 77 (2001), 169187.

[10] J. P. Bès, Invariant manifolds of hypercyclic vectors for the real scalar case, Proc. Amer. Math. Soc. 127 (1999), 1801-1804.

[11] J. P. Bès and A. Peris, Hereditarily hypercyclic operators, J. Funct. Anal. 167 (1999), 94-113.

[12] -, 一, Disjointness in hypercyclicity, J. Math. Anal. Appl., to appear.

[13] C. D. Birkhoff, Démonstration d'un théorème élémentaire sur les fonctions entières, C. R. Acad. Sci. Paris 189 (1929), 473-475.

[14] R. P. Boas, Entire Functions, Academic Press, New York, 1954.

[15] J. Bonet, F. Martínez-Giménez and A. Peris, Linear chaos on Fréchet spaces, Internat. J. Bifur. Chaos Appl. Sci. Engrg. 13 (2003), 1649-1655.

[16] A. Bonilla and K. G. Grosse-Erdmann, Frequently hypercyclic operators and vectors, Ergodic Theory Dynam. Systems 20 (2006), 1-23.

[17] P. S. Bourdon, Invariant manifolds of hypercyclic operators, Proc. Amer. Math. Soc. 118 (1993), 845-847.

[18] P. S. Bourdon and J. H. Shapiro, Cyclic phenomena for composition operators, Mem. Amer. Math. Soc. 596 (1997).

[19] J. B. Conway, Functions of One Complex Variable, Springer, New York, 1986.

[20] G. Costakis and V. Vlachou, Identical approximative sequence for various notions of universality, J. Approx. Theory 132 (2005), 15-24.

[21] S. Dineen, The Schwarz Lemma, Clarendon Press, Oxford, 1989.

[22] D. Gaier, Lectures on Complex Approximation, Birkhäuser, Basel, 1987.

[23] G. Godefroy and J. H. Shapiro, Operators with dense invariant, cyclic vector manifolds, J. Funct. Anal. 98 (1991), 229-269.

[24] S. Grivaux, Construction of operators with a prescribed behaviour, Arch. Math. (Basel) 81 (2003), 291-299.

[25] - , Hypercyclic operators, mixing operators, and the Bounded Steps Problem, J. Operator Theory 54 (2005), 147-168.

[26] K. G. Grosse-Erdmann, Universal families and hypercyclic operators, Bull. Amer. Math. Soc. (N.S.) 36 (1999), 345-381.

[27] —, Hypercyclic and chaotic weighted shifts, Studia Math. 109 (2000), 47-68.

[28] —, Recent developments in hypercyclicity, Rev. R. Acad. Cienc. Ser. A Mat. 97 (2003), 273-286.

[29] M. Heins, On the number of 1-1 directly conformal maps which a multiply-connected plane region of finite connectivity $p(>2)$ admits onto itself, Bull. Amer. Math. Soc. 52 (1946), 454-457.

[30] D. A. Herrero, Limits of hypercyclic and supercyclic operators, J. Funct. Anal. 99 (1991), 179-190.

[31] L. Hörmander, An Introduction to Complex Analysis in Several Variables, NorthHolland, Amsterdam, 1973.

[32] S. G. Krantz, Function Theory of Several Complex Variables, Wiley, New York, 1982 .

[33] G. R. MacLane, Sequences of derivatives and normal families, J. Anal. Math. 2 (1952), 72-87.

[34] F. Martínez-Giménez and A. Peris, Chaos for backward shift operators, Internat. J. Bifur. Chaos Appl. Sci. Engrg. 12 (2002), 1703-1715.

[35] A. Peris and L. Saldivia, Syndetically hypercyclic operators, Integral Equations Operator Theory 51 (2005), 275-281. 
[36] S. Rolewicz, On orbits of elements, Studia Math. 32 (1969), 17-22.

[37] W. Rudin, Real and Complex Analysis, 3rd ed., McGraw-Hill, New York, 1987.

[38] H. Salas, Hypercyclic weighted shifts, Trans. Amer. Math. Soc. 347 (1995), 993-1004.

[39] W. Seidel and J. Walsh, On approximation by Euclidean and non-Euclidean translates of an analytic function, Bull. Amer. Math. Soc. 47 (1941), 916-920.

[40] J. H. Shapiro, Composition Operators and Classical Function Theory, Springer, New York, 1993.

[41] J. Wengenroth, Hypercyclic operators on non-locally convex spaces, Proc. Amer. Math. Soc. 131 (2003), 1759-1761.

Departamento de Análisis Matemático

Facultad de Matemáticas, Apdo. 1160

Avda. Reina Mercedes

41080 Sevilla, Spain

E-mail: lbernal@us.es

Received May 15, 2006

Revised version June 27, 2007 